\title{
Myers-Pospelov Model as an Ensemble of Pais-Uhlenbeck Oscillators: Unitarity and Lorentz Invariance Violation
}

\author{
Justo Lopez-Sarrion \\ Departamento de Física, Universidad de Santiago de Chile, Casilla 307, Santiago, Chile. \\ Carlos M. Reyes \\ Departamento de Ciencias Básicas, Universidad del Bío Bío, Casilla 447, Chillán, Chile.
}

\begin{abstract}
We study a generalization of a Pais-Uhlenbeck oscillator for fermionic variables. Next, we consider an ensemble of these oscillators and we identify a particular case of the Myers-Pospelov model which is relevant for effective theories of quantum gravity. Finally, by taking the advantage of this connection, we analyze, for this model, the unitarity at one loop order in the low energy regime where no ghost states can be created on-shell. This energy regime is the relevant one when we consider the Myers-Pospelov model as a true effective theory coming from new space-time structure.
\end{abstract}

\section{INTRODUCTION}

In recent years, there has been growing interest in quantum field theories containing higher time derivatives. In part because they arise naturally as effective corrections from candidate fundamental theories such as string theory [1], quantum gravity [2] and noncommutative geometry [3]. Moreover, attempts to incorporate such terms have also been given in the context of dark energy [4], Lorentz invariance violation [5]7], radiative corrections [8, 9] perturbative iteration [10] and regularization [11.

Many years ago, a quantum mechanical system with higher time derivatives was proposed by Pais and Uhlenbeck $(\mathrm{P}-\mathrm{U})[12$. This system provides a generalization of the harmonic oscillator by including a fourth time derivative. The first problem that one faces is the unbounded character of the spectrum [13]; sometimes, however, constrained systems can turn around the problem, see [14] and there has been also other proposals [15. There are some redefinitions which avoid these problems but in turn they introduce states of negative norm. Lee and Wick $(\mathrm{L}-\mathrm{W})$ generalize these ideas to relativistic quantum field theories 16. These undefined metric theories have the advantage of being finite in the sense that these higher time derivatives regularize the divergences. The problem of unitarity, which comes out with undefined metric, could be controlled by a prescription due to Cutkosky [17. Recently, these models have been used as an extension of the standard model of particles which fixes the hierarchy problem [18. However, models incorporating operators of mass dimension 5 , which violate Lorentz symmetry, might also contain higher time derivatives. These theories due to Myers and Pospelov (M-P) [6] are supposed to arise from quantum gravity effects at high energies. These two last examples, are part of the increasing interest on higher time derivatives in quantum field theory and, therefore, it is compelling to focus on the technical and physical issues concerning these kind of theories.

In this work, we propose to construct a field theory as ensembles of Pais-Uhlenbeck oscillators in momentum space, instead of harmonic oscillators of the standard relativistic case. We find that this new theory is close related to the Myers-Pospelov fermionic sector. Taking advantage of this relation, we explore the unitarity of the model by modifying the Cutkosky prescription.

All the theories with higher time derivatives seem to have an unavoidable problem because they content negative norm states, at least if there is no gauge symmetry that prevents these modes from appearing in the spectrum [14. The fermonic sector of the Myers-Pospelov model also has this feature, and these ghost modes appear even at tree level in perturbative theory, when the initial state have a total energy greater than the mass of one of these modes. However, this model has to be seen as an effective model, only valid for energies below certain scale, beyond which new physics is described through a more fundamental theory. Hence, lack of unitarity and probabilistic interpretation problems coming from the ghost states are irrelevant because the description of physics beyond that threshold with this model is not justified. Nevertheless, the model must be well defined below the threshold, and it must be capable of giving sensible predictions at this low energy regime. But, unitarity could be spoiled out due to virtual processes even though no on-shell ghosts can be created at low enough energies. This problem was addressed by Cutkosky et al. 17] in the context of relativistic Lee-Wick theories, as mentioned above. They used a convenient prescription to define the propagators in order to keep unitarity order by order in the loop expansion. We will follow the same philosophy to define the propagators in such a way that the Myers-Pospelov model preserves unitarity below the threshold scale. It is worth noting that in the work by Cutkosky the Lorentz invariance is and important ingredient to be considered. However, in our present case, relativistic symmetry only plays a secondary role which will serve to fix part of the prescriptions which connects to the standard relativistic theory at very low energies compared with the threshold.

The layout of this work is the following. In the second section, we review the Pais-Uhlenbeck oscillator and we generalize it to fermions. In the third section, we con- 
struct an ensemble of P-U fermionic oscillator and we identify it as the fermion sector of M-P model. In section four, we analyze the unitarity of the Myers-Pospelov model at one loop order, in the low energy regime by using the structure of P-U oscillators. Finally, we give the conclusions and final comments.

\section{THE ISSUE OF HIGHER TIME DERIVATIVES}

In this section, we introduce the P-U model and we generalize it to the fermionic case which may be less familiar, and hence it will be studied with more details.

\section{A. The Pais-Uhlenbeck model}

The P-U oscillator is, basically, the standard harmonic oscillator with an additional higher time derivative term. To be more precise its equation of motion is

$$
g q^{(4)}+\ddot{q}+\omega^{2} q=0,
$$

where $q^{(4)}$ is a fourth order time derivative and $g$ can be considered a small coupling constant. The equation of motion is obtained from the Lagrangian:

$$
L_{P U}=-\frac{g}{2} \ddot{q}^{2}+\frac{1}{2} \dot{q}^{2}-\frac{1}{2} \omega^{2} q^{2} .
$$

This system can be seen as two standard harmonic oscillators by means of the change of variables,

$$
\begin{aligned}
& q_{+}=\left(\partial_{t}^{2}-k_{-}^{2}\right) q, \\
& q_{-}=\left(\partial_{t}^{2}-k_{+}^{2}\right) q .
\end{aligned}
$$

The Lagrangian with the new variables is

$$
L_{P U}=\frac{1}{2} \dot{q}_{+}^{2}-\frac{1}{2} k_{+}^{2} q_{+}^{2}-\frac{1}{2} \dot{q}_{-}^{2}+\frac{1}{2} k_{-}^{2} q_{-}^{2},
$$

with $k_{ \pm}^{2}=\frac{1}{2 g}\left(1 \mp \sqrt{1-4 g \omega^{2}}\right)$ positive frequencies depending on $g$ and $\omega$. This Lagrangian corresponds to two standard harmonic oscillators with one of them having a relative minus sign respect to the other. This last fact makes a great difference with the simple sum of two oscillators when we add interactions or quantize the theory. Classically, the equations of motion are just the same as two standard harmonic oscillators, however when adding interactions the system becomes unstable due to the unboundedness of the energy. This problem persists under the usual quantization. However, by a redefinition of the vacuum state, the quantum mechanical problem become stable. This last procedure leads to unavoidable negative norm states. These ghosts states could render the theory non unitary when interaction are considered. To be more precise, following the canonical formalism we write the Hamiltonian as:

$$
\hat{H}_{P U}=k_{+} \hat{a}_{+}^{\dagger} \hat{a}_{+}-k_{-} \hat{a}_{-}^{\dagger} \hat{a}_{-}+\frac{1}{2}\left(k_{+}-k_{-}\right),
$$

where $\hat{a}_{+}, \hat{a}_{+}^{\dagger}, \hat{a}_{-}, \hat{a}_{-}^{\dagger}$ are the standard creation and annihilation operators. The second term produces arbitrary negative energy states as can be seen by acting $\hat{a}_{-}^{\dagger}$ on the empty wave function (defined by $\hat{a}_{+} \Phi_{0}=\hat{a}_{-} \Phi_{0}=0$ )

$\Phi_{0}=N \exp \left[-\frac{\sqrt{1-4 g \omega^{2}}}{2\left(k_{+}+k_{-}\right)}\left(k_{+} k_{-} q^{2}+\dot{q}^{2}\right)+\sqrt{-g \omega^{2}} q \dot{q}\right]$.

An alternative proposal to quantize would be to redefine the vacuum $\hat{a}_{+} \Phi_{0}^{\prime}=\hat{a}_{-}^{\dagger} \Phi_{0}^{\prime}=0$. Then the states $\psi_{n}^{\prime}=$ $a_{-}^{(n)} \psi_{0}^{\prime}$ have positive energy, but are ghost states when $n$ is odd. Hence, this procedure stabilizes the theory but it could spoil unitarity when interactions are introduced.

\section{B. Fermionic extension of the P-U model}

Now we consider the fermionic version of the previous model with equation of motion:

$$
g \ddot{\psi}+i \dot{\psi}-\omega \psi=0 .
$$

As before, this is the fermionic harmonic oscillator $(g=$ 0 ) plus a higher derivative term. Without loss of generality we can assume that $\omega$ and $g$ are positive constants.

This equation of motion comes from the Lagrangian

$$
L_{F}=g \bar{\psi} \ddot{\psi}+i \bar{\psi} \dot{\psi}-\omega \bar{\psi} \psi
$$

Let us consider the new variables

$$
\begin{aligned}
& \psi_{-}=\alpha\left(i \partial_{t}-\omega_{+}\right) \psi \\
& \psi_{-}^{\dagger}=\alpha\left(-i \partial_{t}-\omega_{+}\right) \bar{\psi}
\end{aligned}
$$

and

$$
\begin{aligned}
& \psi_{+}=\alpha\left(i \partial_{t}-\omega_{-}\right) \psi \\
& \psi_{+}^{\dagger}=\alpha\left(-i \partial_{t}-\omega_{-}\right) \bar{\psi}
\end{aligned}
$$

where $\alpha=\left(\frac{g}{\omega_{-}-\omega_{+}}\right)^{\frac{1}{2}}$ and the positive frequencies $\omega_{ \pm}$ are defined by

$$
\omega_{ \pm}=\frac{1 \mp \sqrt{1-4 g \omega}}{2 g}
$$

where $\omega_{-}>\omega_{+}>0$. The Lagrangian in terms of this new variables is

$$
L=\psi_{+}^{\dagger}\left(i \partial_{t}-\omega_{+}\right) \psi_{+}-\psi_{-}^{\dagger}\left(i \partial_{t}-\omega_{-}\right) \psi_{-},
$$

which can be reduced to the original Lagrangian (8), by replacing Eqs. (9) and 10 on it.

Now, the usual variation of the above Lagrangian gives the equations of motion

$$
\begin{aligned}
& \left(i \partial_{t}-\omega_{+}\right) \psi_{+}=0 \\
& \left(i \partial_{t}-\omega_{-}\right) \psi_{-}=0 .
\end{aligned}
$$

As in the bosonic case the system is decoupled into two standard oscillators, but with a global minus sign for the 
$\psi_{-}$. Following the canonical quantization for fermions, one can check that the canonical conjugate momentum for $\psi_{+}$is $i \psi_{+}^{\dagger}$, but for $\psi_{-}$is $-i \psi_{-}^{\dagger}$. Hence, this gives the anticommutators in the new variables

$$
\begin{aligned}
& \left\{\psi_{+}, \psi_{+}^{\dagger}\right\}=1, \\
& \left\{\psi_{-}, \psi_{-}^{\dagger}\right\}=-1 .
\end{aligned}
$$

Then, the operators $\psi_{-}, \psi_{-}^{\dagger}, \psi_{+}$and $\psi_{+}^{\dagger}$ are standard creation and annihilation operators, respectively, but $\psi_{-}$ and $\psi_{-}^{\dagger}$ create and annihilate states of negative norm.

The original variables can be written in terms of these operators as

$$
\begin{aligned}
\psi & =\frac{\psi_{-}-\psi_{+}}{(1-4 g \omega)^{1 / 4}}, \\
\dot{\psi} & =-i \frac{\omega_{-} \psi_{-}-\omega_{+} \psi_{+}}{(1-4 g \omega)^{1 / 4}},
\end{aligned}
$$

so the Hamiltonian turns out to be

$$
H_{F}=\omega_{+} \psi_{+}^{\dagger} \psi_{+}-\omega_{-} \psi_{-}^{\dagger} \psi_{-} .
$$

This expression can be compared to the one for the $\mathrm{P}$ $\mathrm{U}$ Hamiltonian. They are the same expression, except for an irrelevant constant. However, this system corresponds to a four state system, and hence, the spectrum is bounded below, and the vacuum is normalizable. In fact, the true vacuum is the state annihilated by $\psi_{-}$and $\psi_{+}$, namely, $\psi_{-} \Phi_{0}=\psi_{+} \Phi_{0}=0$ where the explicit form of the wave function for the vacuum has been calculated in the appendix.

Moreover, the rest of the states are $\Phi_{1}=\psi_{+}^{\dagger} \Phi_{0}$, with energy $\omega_{+}, \Phi_{2}=\psi_{-}^{\dagger} \Phi_{0}$ with energy $\omega_{-}$and $\Phi_{3}=$ $\psi_{-}^{\dagger} \psi_{+}^{\dagger} \Phi_{0}$ with energy $\omega_{+}+\omega_{-}$. Nevertheless, the $\Phi_{2}$ and $\Phi_{3}$ operator correspond to ghost states. In the appendix we follow the Schröedinger quantization procedure and the interpretation is the same as above.

Therefore, in the fermionic case we just have one alternative to quantize the theory, in contrast with the bosonic case where we can either have unstable spectrum or indefinite norms in agreement with the analysis with Lee and Wick 16.

Finally, we calculate the temporal ordered Green's functions which will be useful in later perturbative considerations. To do this, let us consider the usual definition

$$
\begin{aligned}
G_{a b}^{F}(t) & =\theta(t)\left\langle 0\left|\psi_{a}(t) \psi_{b}^{\dagger}(0)\right| 0\right\rangle-\theta(-t)\left\langle 0\left|\psi_{b}^{\dagger}(0) \psi_{a}(t)\right| 0\right\rangle \\
& =\left\langle 0\left|\mathcal{T}\left(\psi_{a}(t) \psi_{b}^{\dagger}(0)\right)\right| 0\right\rangle
\end{aligned}
$$

where $a, b= \pm$. By solving the equations of motion (13) we have

$$
\psi_{a}(t)=e^{-i \omega_{a} t} \psi_{a}(0) .
$$

Replacing this in the Green function expression and taking into account that $\psi_{a}(0)|0\rangle=0$ we arrive at

$$
G_{a b}^{F}(t)=a e^{-i \omega_{a} t} \theta(t) \delta_{a b},
$$

where we have made use of the anticommutation relations (14). This Green function satisfies the equation

$$
\left(i \partial_{t}-\omega_{a}\right) G_{a b}^{F}(t)=a i \delta(t) \delta_{a b}
$$

We can use an integral representation with the following prescription

$$
G_{a b}^{F}(t)=a i \delta_{a b} \int \frac{d \omega}{2 \pi} \frac{e^{-i \omega t}}{\omega-\omega_{a}+i \epsilon},
$$

where the prescription $\epsilon>0$ is used.

Note that we are dealing with a $(0+1)$ - dimension model where there is no particles and antiparticles. In a more realistic $(3+1)$ - dimension case, particles and antiparticles are present, and the usual prescription for $\epsilon$ is positive for particles and negative for antiparticles.

\section{FERMIONIC PAIS-UHLENBECK ENSEMBLE AND THE MYERS-POSPELOV MODEL}

In this section we propose a generalization of the fermionic P-U model to a quantum field theory. This generalization may be performed in different ways. However, we will use the following approach: we consider the standard field theory as an ensemble of decoupled harmonic oscillators in the momentum space, with and additional term containing higher time derivatives, as we did in the previous section.

\section{A. Covariant free field theory as ensembles of harmonic oscillators}

To fix ideas, let us review how we can go from the standard bosonic and fermionic harmonic oscillator, to a scalar and Dirac free field theories, respectively.

First, we consider an ensemble of bosonic harmonic oscillators whose dynamical variables are labeled by a vector $\vec{p}$ with frequency $\omega_{\vec{p}}$, so that the Lagrangian of this ensemble is a sum of the Lagrangian of the individual oscillators over all possible values of $\vec{p}$. In the continuum limit the sum becomes an integral, and we can write the Lagrangian as

$$
L=-\frac{1}{2} \int d^{3} p \phi_{\vec{p}}^{\dagger}\left(\partial_{t}^{2}+\omega_{\vec{p}}^{2}\right) \phi_{\vec{p}}
$$

where $\phi_{\vec{p}}$ is the dynamical variable of the oscillator. In order to make contact with a relativistic theory we assume that $\omega_{\vec{p}}=\sqrt{\vec{p}^{2}+m^{2}}$. After a Fourier transform we end up with the free scalar field theory

$$
L=-\frac{1}{2} \int d^{3} x \phi_{\vec{x}}\left(\square+m^{2}\right) \phi_{\vec{x}},
$$

where $\phi_{\vec{x}}(t)$ is the Fourier transform of $\phi_{\vec{p}}(t)$.

The fermionic case is less direct. In order to recover the free Dirac field, we will need four fermionic oscillators 
at each point in the $\vec{p}$ vector space. We will label this fermion variables as $\psi_{i \vec{p}}^{s}$ where $s$ will be the spinor index and $i$ will specify particle or antiparticle modes. Then, the Lagrangian at each point $\vec{p}$ is

$$
L_{\vec{p}}=\sum_{s, i} \psi_{i \vec{p}}^{\dagger s}\left(i \partial_{t}-\omega_{i}^{s}(\vec{p})\right) \psi_{i \vec{p}}^{s}
$$

Again, the ensemble of this oscillators will be given through the integral over all possible values of $\vec{p}$. To get the relativistic theory, we assume that the frequencies have the form

$$
\omega_{i}^{s}(\vec{p})=\epsilon_{i} \omega_{\vec{p}}
$$

with $\epsilon_{i}= \pm 1$ for particles and antiparticles, respectively, and we write the new variables

$$
\psi_{\vec{p}}(t)=\frac{1}{\sqrt{2 \omega_{\vec{p}}}} \sum_{s, i} w_{i}^{s}(\vec{p}) \psi_{i \vec{p}}^{s}(t),
$$

where $w_{1}^{s}(\vec{p})=u^{s}(\vec{p})$ is the amplitude of the free wave function that is solution of the Dirac equation for particles of spin $s$ and momentum $\vec{p}$ and $w_{2}^{s}(\vec{p})=v^{s}(-\vec{p})$ the free wave function for antiparticles of spin $s$ and momentum $-\vec{p}$. In terms of these basis and making use of the orthogonality relation

$$
w_{i}^{\dagger r}(\vec{p}) w_{j}^{s}(\vec{p})=2 \omega_{\vec{p}} \delta^{r s} \delta^{i j}
$$

and the completeness relations

$$
\sum_{s} w_{i}^{s}(\vec{p}) w_{i}^{s \dagger}(\vec{p})=\omega_{\vec{p}}+\epsilon_{i} h_{D}(\vec{p}),
$$

where $h_{D}(\vec{p})=\vec{\alpha} \cdot \vec{p}+m \beta$ and $\vec{\alpha}=\gamma^{0} \vec{\gamma}$ and $\beta=\gamma^{0}$ are the $4 \times 4$ Hermitian Dirac matrices, the Lagrangian turns out to be

$$
L_{\vec{p}}=\psi_{\vec{p}}^{\dagger}\left(i \partial_{t}-h_{D}(\vec{p}) \psi_{\vec{p}}\right.
$$

Hence, the Lagrangian of the ensemble, which is the integral of the above expression, can be written in terms of the Fourier transform variables $\psi_{\vec{x}}$ and $\bar{\psi}_{\vec{x}}=\psi_{\vec{x}}^{\dagger} \beta$ as

$$
L=\int \frac{d^{3} p}{(2 \pi)^{3}} L_{\vec{p}}=\int d^{3} x \bar{\psi}_{\vec{x}}(i \partial \cdot \gamma-m) \psi_{\vec{x}} .
$$

Summarizing, this procedure shows us that given an ensemble of oscillators, with frequencies depending on free parameters, we can find a space that we call $x$-space where the ensemble is described as a relativistic standard free field theory.

\section{B. An ensemble of P-U harmonic oscillators: Fermionic Myers-Pospelov model}

Now, we come to the main part of the section. As it was mentioned before, we will consider an ensemble of $\mathrm{P}-\mathrm{U}$ harmonic oscillators and see what theory produces.
We set out from Eq. 24 and 25, but we add a higher time derivative term

$$
L_{\vec{p}}=\sum_{s, i} \psi_{i \vec{p}}^{\dagger s}\left(g \partial_{t}^{2}+i \partial_{t}-\epsilon_{i} \omega_{\vec{p}}\right) \psi_{i \vec{p}}^{s}
$$

Then we consider the variable $\psi_{\vec{p}}$ as defined in Eq. 26. and performing a Fourier transform the Lagrangian has the form

$$
L=\int \frac{d^{3} p}{(2 \pi)^{3}} L_{\vec{p}}=\int d^{3} x \bar{\psi}_{\vec{x}}\left(i \partial \cdot \gamma+g \partial_{t}^{2}-m\right) \psi_{\vec{x}}
$$

This model, arising from an ensemble of $\mathrm{P}-\mathrm{U}$ harmonic oscillators, can be identified with fermionic sector of the well known M-P model, which is an effective Lorentz invariance violating field theory, containing quantum gravity effects. To clarify this claim, we introduce a vector $n=(1,0,0,0)$, and the former Lagrangian can be written as

$$
L=\int d^{3} x \bar{\psi}_{\vec{x}}\left(i \not \partial+g h(n \cdot \partial)^{2}-m\right) \psi_{\vec{x}}
$$

This is the Myers and Pospelov fermionic sector with $g_{2}=0$ and an isotropic background with 5-dimension operators. This model has been widely studied in the context of quantum gravity effects [6, 19].

\section{UNITARITY}

Indefinite metric theories have potential physical problems which may spoil out any predictive ability and even worse, they would lack of a sensible physical interpretation [20]. The source of these problems is the failure of the unitarity of the time evolution operator. Many years ago, Cutkosky proposed a prescription to keep the unitarity at perturbative level for nonhermitian but Lorentz symmetric theories [16. This procedure was successfully performed for the so called Lee-Wick models.

In this section, we attempt to fit similar prescription in order to prove the unitarity for the M-P fermionic sector at one loop perturbative level, and for energies less than the ultraviolet scale of the theory. This modification must take into account the Lorentz invariance violation intrinsic in the M-P model. As it was mentioned in the introduction, we are only interested in this low energy regime because it is the relevant one for a theory which must be seen as an effective theory parametrizing new features of space-time structure which may come from a more fundamental theory.

The first thing to do is to write down the relevant propagators. In the M-P theory the propagators, in terms of fundamental fields are complicated, and it is more convenient to express them in terms of the P-U modes. These are the P-U modes

$$
\psi^{r, i}(t, \vec{p}) \equiv \frac{1}{\sqrt{2 \omega_{\vec{p}}}} w_{i}^{r \dagger}(\vec{p}) \psi(t, \vec{p})
$$




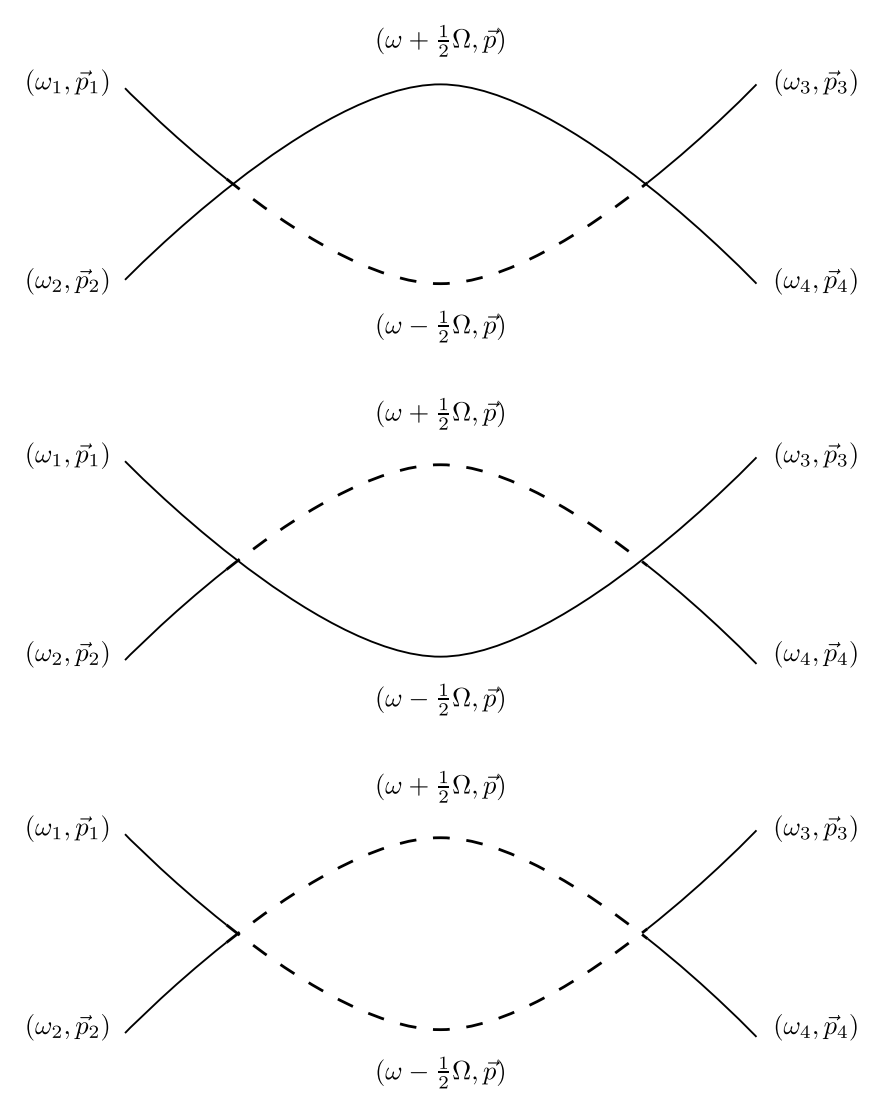

FIG. 1. We show the diagrams that contain ghosts and therefore those that can spoil unitarity at one loop order. In the first figure we show the diagram corresponding to the amplitude $M_{+-}$, in the second, the amplitude $M_{-+}$, and in the last figure the amplitude corresponding to $M_{--}$.

in terms of the Fourier transform fields

$\psi(t, \vec{p})=\int d^{3} x \psi(t, \vec{x}) e^{i \vec{p} \cdot \vec{x}}$. Now these new variables correspond to four P-U oscillators with frequency $\omega(\vec{p})=$ $\epsilon_{i} \omega_{\vec{p}}$. As we have seen in section II this oscillators are equivalent to two fermionic oscillators with frequencies

$$
\omega_{ \pm i}^{r}(\vec{p}) \equiv \frac{1 \mp \sqrt{1-4 g \epsilon_{i} \omega_{\vec{p}}}}{2 g},
$$

for each P-U oscillators labeled with $i$ and $r$. The \pm signs refers to the signs of the norm for those modes. Now, it is straightforward to calculate the propagators,

$$
G_{F a b}^{r s, i j}(t, \vec{x}) \equiv\langle 0| \mathcal{T}\left(\psi_{i a}^{r}(t, \vec{x}) \psi_{j b}^{s \dagger}(0)|0\rangle,\right.
$$

corresponding to these modes by looking at the expression at the end of the section II. Namely the Fourier transform of this propagators are,

$$
G_{F a b}^{r s, i j}(\omega, \vec{p})=a \frac{i}{2 \omega_{\vec{p}}} \times \frac{w_{i}^{r}(\vec{p}) w_{j}^{s \dagger}(\vec{p})}{\omega-\omega_{a i}^{r}(\vec{p})+i \delta_{i}^{a}} \delta_{a b},
$$

where $\delta_{i}^{a}$ will correspond to some suitable prescription to fit later. In order to recover the standard Lorentz symmetric limit we must impose the prescription $\delta_{1}^{+}=$ $-\delta_{2}^{+}=\epsilon>0$. To see this, the standard Feynman propagator can be related with the $\mathrm{P}-\mathrm{U}$ propagators by

$$
\Delta_{F}(\omega, \vec{p})=\sum_{s, i} G_{F++}^{s s, i i}(\omega, \vec{p}) \gamma^{0} .
$$

Then, in the limit $g \rightarrow 0$ we have $\omega_{+i}^{s}(\vec{p}) \rightarrow \epsilon_{i} \omega_{\vec{p}}$, and making use of the completeness relations (28) we obtain the standard relativistic expression

$$
\begin{aligned}
\Delta_{F}(\vec{p}) & =\frac{i}{2 \omega_{\vec{p}}}\left[\frac{\omega_{\vec{p}}+h_{D}(\vec{p})}{\omega-\omega_{+1}(\vec{p})+i \epsilon}+\frac{\omega_{\vec{p}}-h_{D}(\vec{p})}{\omega-\omega_{+2}(\vec{p})-i \epsilon}\right] \gamma^{0} \\
& \rightarrow \frac{i(\not p+m)}{p^{2}-m^{2}+i \epsilon} .
\end{aligned}
$$

Now, we will prove that with a suitable prescription for $\delta_{i}^{-}$we can keep the unitarity of the theory at one loop perturbation, at least. Let us write the evolution operator in terms of the scattering matrix,

$$
\mathcal{U}=1+i S
$$

Then, the unitarity implies,

$$
\mathcal{U U}^{\dagger}=1+i\left(S-S^{\dagger}\right)+S S^{\dagger}=1 .
$$

Namely, we must prove that $-i\left(S-S^{\dagger}\right)=S S^{\dagger}$.

Perturbatively, we must show that

$$
2 \operatorname{Im} \mathcal{M}(\text { in } \mid \text { out })=\sum_{\text {Phys }} \mathcal{M}(\text { in } \mid \text { Phys }) \mathcal{M}(\text { Phys } \mid \text { out }),(42)
$$

where $\mathcal{M}$ are the perturbative elements of the scattering matrix and the sum is over all possible physical states allowed by the conservation of energy and momentum.

We are interested in showing this equality for in and out states for particles with energies less than the ultraviolet scale $\frac{1}{4 g}$. At tree level, due to the energy and momentum conservation, it is impossible to create ghosts states and then, the contribution is zero at both sides of the previous equation, at first order in perturbation correction. The first chance to break down the equation (42) is at one loop order; however, for the same reason above mentioned, the right hand side of equation 42 is zero. Then, we must check that the imaginary part of $\mathcal{M}$ is zero at the first radiative correction.

It is sufficient to analyze the integrals corresponding to the diagrams in the Fig. 1, which have problematic ghost internal lines. To simplify, we will work in the center of mass frame, with total energy $\Omega$ and total momentum $\vec{P}=0$. The integrals contributing to this graphs are,

$$
\begin{array}{r}
\mathcal{M}_{a b}(\Omega)=\frac{\alpha^{2}}{2} \sum_{\substack{r, s, r^{\prime}, s^{\prime} \\
i, j, i^{\prime}, j^{\prime}}} \int d \omega d^{3} p \\
\operatorname{tr}\left(G_{F a a}^{r s, i j}(\omega-\Omega / 2, \vec{p}) G_{F b b}^{r^{\prime} s^{\prime}, i^{\prime} j^{\prime}}(\omega+\Omega / 2, \vec{p})\right)
\end{array}
$$

where $\alpha$ is the coupling constant which introduces an interaction and $a, b= \pm$. 
By using the orthogonality property in the Eq. 27) the sum of the integrands of these diagrams are proportional to the functions,

$$
\begin{array}{r}
\Gamma_{a b}(\omega)=\sum_{i} \frac{1}{\omega-\Omega / 2-\omega_{a i}(\vec{p})+i \delta_{i}^{a}} \times \\
\frac{1}{\omega+\Omega / 2-\omega_{b i}(\vec{p})+i \delta_{i}^{b}}
\end{array}
$$

The pole structure of these integrands is shown in Fig. 2 and Fig. 3, and their residues at the poles are

$$
\begin{aligned}
& \underset{\omega_{a i}-\Omega / 2}{\operatorname{Res}} \Gamma_{a b}=\frac{1}{\omega_{a i}-\omega_{b i}-\Omega}, \\
& \operatorname{Res}_{\omega_{b i}+\Omega / 2} \Gamma_{a b}=\frac{1}{\omega_{b i}-\omega_{a i}+\Omega} .
\end{aligned}
$$

Any prescription to avoid the poles is equivalent to a path integration in the $\omega$-plane $\mathcal{C}$.

For $\omega_{\vec{p}}<\frac{1}{4 q}$, we choose the prescription $\delta_{1}^{-}=-\delta_{2}^{-}=$ $\delta>0$. When we integrate $\omega$ over the real axis, this prescription leave the poles $1^{-}$below and $2^{-}$above. This is equivalent to the contour $\mathcal{C}$ shown in Fig. 2. The conjugate of this integration is given by the contour $\mathcal{C}^{*}$. Hence, the imaginary part of the integral is proportional to the closed contour integration over $\mathcal{C}-\mathcal{C}^{*}$, which is proportional to the sum of the residues.

Thus, for $\operatorname{Im} \mathcal{M}_{--}$we have,

$$
\begin{aligned}
\oint_{\mathcal{C}-\mathcal{C}^{*}} \Gamma_{--} d \omega & \propto \sum_{\text {Poles }} \epsilon_{i} \operatorname{Res} \Gamma_{-i}(\omega) \\
& =\sum_{i} \epsilon_{i}\left(\frac{1}{-\Omega}+\frac{1}{\Omega}\right)=0 .
\end{aligned}
$$

And for $\operatorname{Im}\left(\mathcal{M}_{+-}+\mathcal{M}_{-+}\right)$, we have

$$
\begin{aligned}
\oint_{\mathcal{C}-\mathcal{C}^{*}} & \left(\Gamma_{+-}+\Gamma_{+-}\right) d \omega \propto \sum_{\text {Poles }} \epsilon_{ \pm i} \epsilon_{i \Omega / 2} \operatorname{Res}\left(\Gamma_{+-}+\Gamma_{-+}\right), \\
= & \sum_{i} \epsilon_{i}\left(\frac{1}{\omega_{+i}-\omega_{-i}-\Omega}+\frac{1}{\omega_{-i}-\omega_{+i}+\Omega}\right)=0,
\end{aligned}
$$

where $\epsilon_{i}$ stands for signs \pm 1 of the closed contours orientation that surround the poles.

For internal momentum above the critical scale, $\omega_{\vec{p}}>$ $\frac{1}{4 g}$, some poles become complex, as it can be seen in Fig. 3 . Now the integral over the real axis of $\omega$ can be closed around the lower half-plane, picking up the residues at the poles in this half-plane. The complex conjugate integration will be closed above the upper-half plane, picking up now the poles in this half-plane. Thus, the imaginary part of the integral with complex poles is given by a contour enclosing these poles. This prescription is equivalent to take the poles corresponding to $1^{+}$moving up and the ones corresponding to $1^{-}$moving down. Then, it can be seen that the prescription given above corresponds to take the integration path $\mathcal{C}$ and $\mathcal{C}^{*}$ shown in Fig. 3, which are smooth deformations of those paths in Fig. 2.

Now, the closed contour integrals take the residues in the same way as before, and their sum vanishes again.

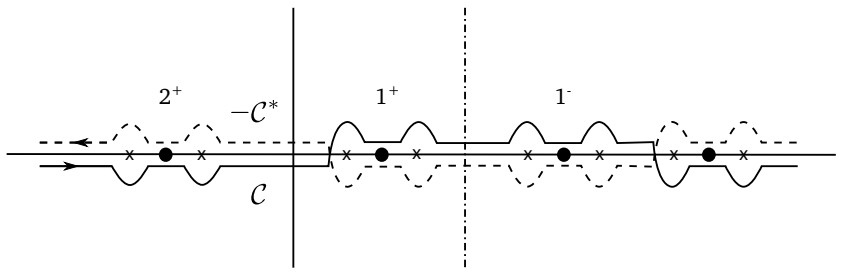

FIG. 2. We show the pole structure for $\omega_{\vec{p}}<1 / 4 g$, the notation $i^{ \pm}$stands for particles and antiparticles with positive or negative norms $( \pm)$. The crosses correspond to the shifted poles in the integrals of the amplitudes at one loop order. The contour $C$ corresponds to the prescription described in the text, $C^{*}$ to its conjugate. The vertical line corresponds to the critical region above which the poles become complex.

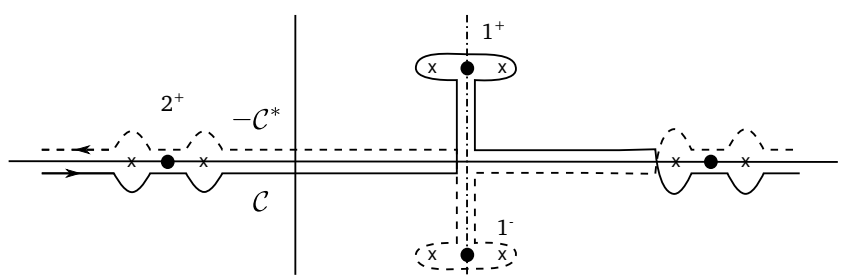

FIG. 3. The pole structure above the critical region $\omega_{\vec{p}}>$ $1 / 4 g$. The poles corresponding to $1^{+}$and $1^{-}$collapse to each other and they become complex. According to our prescription the pole $1^{+}$go up and $1^{-}$go down in the critical vertical line. The integration contours $C$ and $C^{*}$ are smooth deformation of those of the Fig. 2.

Thus, we have found a particular prescription to maintain the unitarity at one loop order.

We have used a somehow arbitrary interaction, however, the procedure followed above can work with other general interactions.

Finally, let us note that there is some ambiguity in choosing the prescription which keep the unitarity. This freedom could be used to maintain the unitarity at higher radiative corrections. Nevertheless, this is far from being clear in this analysis.

\section{FINAL COMMENTS}

Summarizing, in this work we have found a connection between an emsemble of fermionic P-U oscillators, and the well known M-P model in the fermionic sector. This fact has allowed us to prove the unitarity of the M-P fermonic sector at one loop order in perturbation theory at low energies where the theory is applicable, even though, it contains higher time derivative terms. This statement is the most important result in this study, because it permits to have sensible predictions and physical interpretations.

Our study shows that for Lorentz invariant violating theories with higher time derivative terms - with fermions - it is possible to maintain the unitarity at first order in radiative corrections. 
The prove of the unitarity at higher radiative corrections, is left as a future work. Also, it would be interesting to do phenomenological predictions from this theory.

\section{ACKNOWLEDGMENTS}

We thank T. Fernandez and Prof. D. Restrepo for helpful support during the preparation of the manuscript. J.L-S. acknowledges support from DICYT Grant No. 041131LS (USACH) and FONDECYT-Chile Grant No. 1100777 and wants to thank the hospitality at the Universidad de Antoquia. C. M. R. acknowledges partial support from Dirección de Investigación de la Universidad del Bío-Bío (DIUBB) Grant No. 123809 3/R.

\section{Appendix A: Schröedinger Quantization of the P-U Fermionic Oscillator}

In this appendix we show an alternative procedure to quantize the P-U fermionic oscillator. This formalism gives us an explicit form of the vacuum state, and shows how the negative norm states appear.

The following Hamiltonian

$$
H=-g \dot{\bar{\psi}} \dot{\psi}+\omega \bar{\psi} \psi
$$

and the non vanishing anticommutators

$$
\{\dot{\bar{\psi}}, \psi\}=-\frac{i}{g}, \quad\{\dot{\psi}, \bar{\psi}\}=\frac{i}{g}, \quad\{\dot{\psi}, \dot{\bar{\psi}}\}=-\frac{1}{g^{2}}
$$

reproduce the fermionic $\mathrm{P}-\mathrm{U}$ equations of motion presented in section II.

Using the Schröedinger representation in terms of
Grassman variables and their derivatives, we have,

$$
\begin{aligned}
\dot{\bar{\psi}} & =-\frac{i}{g} \frac{\partial}{\partial \psi}+\frac{i}{2 g} \bar{\psi}, \\
\dot{\psi} & =\frac{i}{g} \frac{\partial}{\partial \bar{\psi}}-\frac{i}{2 g} \psi
\end{aligned}
$$

And the Hamiltonian in this representation is,

$$
\begin{aligned}
: H: & =-\frac{1}{g} \frac{\partial}{\partial \psi} \frac{\partial}{\partial \bar{\psi}}+\omega\left(1-\frac{1}{4 g \omega}\right) \bar{\psi} \psi \\
& +\frac{1}{2 g}\left(\bar{\psi} \frac{\partial}{\partial \bar{\psi}}-\psi \frac{\partial}{\partial \psi}\right)+\frac{1}{2 g} .
\end{aligned}
$$

The first and second lines of this equation commute to each other, so it is very easy to find the eigenfunctions,

$$
\begin{array}{ll}
\Phi_{0}=\psi, & E_{0}=0, \\
\Phi_{1}=\frac{\sqrt{2}}{(1-4 g \omega)^{\frac{1}{4}}} e^{\frac{\sqrt{1-4 g \omega}}{2} \bar{\psi} \psi,} & E_{1}=\omega_{+}, \\
\Phi_{2}=\frac{\sqrt{2}}{(1-4 g \omega)^{\frac{1}{4}}} e^{-\frac{\sqrt{1-4 g \omega}}{2} \bar{\psi} \psi,} & E_{2}=\omega_{-}, \\
\Phi_{3}=\bar{\psi}, & E_{3}=\omega_{+}+\omega_{-},
\end{array}
$$

where $\omega_{ \pm}$are defined in section II. For positive $g$, the state $\Phi_{0}$ corresponds to the lowest energy state, and then it represents the vacuum of the theory. Now, it is clear, that this is a four state system with bounded energies.

Regarding the normalization, we can define the Berezin measure as $\int d \bar{\psi} d \psi \bar{\psi} \psi=+1$, the scalar product of wave functions $F(\psi, \bar{\psi})$ and $G(\psi, \bar{\psi})$ is

$$
\langle G \mid F\rangle \equiv \int d \bar{\psi} d \psi \bar{G} F .
$$

Then we can see that

$$
\left\langle\Phi_{0} \mid \Phi_{0}\right\rangle=\left\langle\Phi_{1} \mid \Phi_{1}\right\rangle=1
$$

However,

$$
\left\langle\Phi_{2} \mid \Phi_{2}\right\rangle=\left\langle\Phi_{3} \mid \Phi_{3}\right\rangle=-1
$$

are states with negative norm. This agrees with the results in section II.
[1] D. A. Eliezer, R. P. Woodard, Nucl. Phys. B325, 389 (1989).

[2] K. S. Stelle, Phys. Rev. D16, 953-969 (1977); E. T. Tomboulis, Phys. Rev. Lett. 52, 1173 (1984).

[3] Z. Guralnik, R. Jackiw, S. Y. Pi and A. P. Polychronakos, Phys. Lett. B 517, 450 (2001).

[4] R. P. Woodard, Lect. Notes Phys. 720, 403-433 (2007).

[5] V. A. Kostelecky and M. Mewes, Phys. Rev. D 80, 015020 (2009); A. Kostelecky and M. Mewes, Phys. Rev. D 85, 096005 (2012).

[6] R. C. Myers, M. Pospelov, Phys. Rev. Lett. 90, 211601 (2003).

[7] P. A. Bolokhov and M. Pospelov, Phys. Rev. D 77, 025022 (2008).
[8] I. Antoniadis, E. Dudas and D. M. Ghilencea, Nucl. Phys. B 767, 29 (2007); D. Anselmi, Eur. Phys. J. C 65, 523 (2010).

[9] T. Mariz, J. R. Nascimento and A. Y. .Petrov, Phys. Rev. D 85, 125003 (2012); T. Mariz, Phys. Rev. D 83, 045018 (2011).

[10] X. Jaen, J. Llosa, A. Molina, Phys. Rev. D34, 2302 (1986).

[11] Thirring W., Phys. Rev. 77 (1950), 570; M. Visser, Phys. Rev. D 80, 025011 (2009).

[12] A. Pais, G. E. Uhlenbeck, Phys. Rev. 79, 145-165 (1950).

[13] Ostrogradsky M., Mem. Acad. St. Petersbourg VI 4 (1850), 385-517.

[14] M. S. Plyushchay, Mod. Phys. Lett. A 4, 837 (1989); 
A. V. Smilga, Nucl. Phys. B706, 598-614 (2005).

[15] S. W. Hawking, T. Hertog, Phys. Rev. D65, 103515 (2002); C. M. Bender, P. D. Mannheim, Phys. Rev. Lett. 100, 110402 (2008); A. Dector, H. A. Morales-Tecotl, L. F. Urrutia, J. D. Vergara, SIGMA 5, 053 (2009).

[16] T. D. Lee and G. C. Wick, Nucl. Phys. B 9, 209 (1969); T. D. Lee, G. C. Wick, Phys. Rev. D2, 1033-1048 (1970).

[17] R. E. Cutkosky, P. V. Landshoff, D. I. Olive, J. C. Polkinghorne, Nucl. Phys. B12, 281-300 (1969).

[18] J. R. Espinosa, B. Grinstein, D. O'Connell, M. B. Wise,
Phys. Rev. D77, 085002 (2008); B. Grinstein, D. O'Connell, M. B. Wise, Phys. Rev. D77, 025012 (2008); B. Fornal, B. Grinstein and M. B. Wise, Phys. Lett. B 674, 330 (2009).

[19] J. Lopez-Sarrion and C. M. Reyes, Eur. Phys. J. C. 72, 2150 (2012).

[20] A. Mostafazadeh, J. Math. Phys. 44, 974 (2003); A. Das and L. Greenwood, Phys. Lett. B678, 504 (2009). 